\title{
The Application of Commercial Calculating Machines to Scientific Computing
}

I propose to deal with one era in computing, but will first recall some of the landmarks in that art. Perhaps the first was the introduction of the decimal system. Then came the invention or discovery of logarithms in 1614 by NAPIER, which enabled multiplications and divisions to be done much more rapidly than had been the case before. Briggs' industry in tabulating logarithms of numbers and of trigonometrical functions made Napier's discovery immediately available to all computers.

Although PASCAL constructed the first calculating machine in 1642, and although Leibniz, Morland, Stanhope and others contributed ideas and designs, nearly two centuries were to elapse before a commercial calculating machine could be purchased. This was the arithmometer of ThOMAS DE Colmar, first used in actuarial offices in Paris in the 1820's. It uses the stopped-wheel principle enunciated by Leibnitz to determine which digit shall be added in each columnar position. There is little to report till about half a century later, when ODHNER invented his pin-wheel for conveying varying digits to the machine. This principle is still used in the Swedish Odhner and other barrel-type crank-driven hand machines, particularly the German Brunsviga, which acquired manufacturing rights about 1890 . This marked the beginning of a period of development, which continued with ever-growing intensity till, for the second time this century, progress was interrupted by war. These developments include keyboard setting, electric drive, automatic control of multiplication and division, duplication of registers, and storage and transfer facilities.

Although these machines were developed primarily for the wide fields of commercial and accounting application, they lend themselves to mathematical and scientific computing, which consists, after all, of the four fundamental processes of addition, subtraction, multiplication and division - just the processes that any calculating machine is designed to perform. Between the two world wars comes the era under review-that in which scientists discovered more and more how numerical processes could be lightened by mass-produced commercial machines. To make such a discovery, it is necessary that one person should understand both the machine and the problem. As far as possible commercial machines should be used in their standard form, although minor alterations are permissible. The computer's art lies mainly in his ability to manipulate the problem and to apply ingenuity and low cunning in developing techniques that take advantage of the mechanical features of the machine.

If I express the view that this process of adaptation has gone further in Great Britain than in U. S. A., I should like to give a rational explanation of why this should be so. Our great advantage is that we have more material with which to operate. In U.S. A. there are only three outstanding crankdriven calculating machines-the Marchant, Monroe and Friden. These are similar in capacity and in possessing keyboard setting, electric drive, automatic multiplication and division, but few special features such as one finds in the German Brunsviga, Mercedes, Archimedes and Rheinmetall, or the 
Swiss Madas, or the Swedish Facit. Perhaps a contributing factor has been the European willingness to use a large proportion of hand machines, for hand machines of ten have facilities and flexibility not possessed to the same degree by their more affluent electric cousins. I shall revert later to this theme in more detail, and mention some special features of machines and some special techniques.

The adaptation age has overlapped the beginning of another-namely that of the construction of special machines. I have sometimes felt that physicists and engineers are too prone to ask themselves "What physical, mechanical or electrical analogue can I find to the equation I have to solve?" and rush to the drawing board and lathe before enquiring whether any of the many machines that can be purchased over the counter will not do the job. Too of ten the only tangible result has been a pile of blue prints, and perhaps. a single machine that reaches old age with teething troubles-a machine that benefits only a few people and often impoverishes the inventor, who pays for patents that he cannot sell, for modern manufacturers think in terms of thousands of machines. I do not for a moment contend that there should not be any special design or development, but I do urge that this should be preceded by an exhaustive attempt to utilise what exists, and that new development is proper only when what we now have leaves much to be desired, or when quantity or time considerations, or the relative cost of labour and machinery, point a definite finger. What would not be right in India, where subsistence requirements may almost be measured in handfuls of rice, can easily be justified if computers cost $\$ 2000$ to $\$ 3000$ a year plus supervision, administration and costly accommodation. In other words look at what has been provided by and for industry before you leap to the adventure of special design.

Outstanding among special developments is one for which there can be nothing but praise, namely differential analysers. Although differential equations can be (and are) solved by finite difference methods on existing machines, the quantity of low-accuracy solutions required today is such that time and cost would be prohibitive. The use of machines for handling infinitesimals rather than finite quantities has fully justified itself, and we may all feel grateful to VANNEVAR BUSH, whose pioneer work has been an inspiration to many followers.

We are now living in an age of development of relay and electronic computers designed to perform calculations on a scale and with a speed undreamt of a decade ago. Many of these still have to face their real life tests. They have been brought into being under the stress of military expediency and urgency. Some have arrived (or are arriving) too late for the war that is now happily concluded, and it remains to be seen whether future research can absorb their enormous output capacity at an economic level. It is only fair that the need for new machines should be judged, not in the light of what we now do, but in terms of what we could profitably do if they were available. In many cases they will enable numerical mathematical analysis, with its wide range of choice in parameters, to replace experiments with costly models.

To revert to our title. The prejudice against hand machines, or should we say the disposition to use electric machines, which is such a strong feature of American usage, is far less pronounced in Europe. Whilst this arises 
partly from economic considerations, a contributing factor is the wider range of possibilities and flexibility provided. This is well illustrated by the Brunsviga, a pre-war favourite among scientists, especially for private exploratory work. Its outstanding feature (1925) is a device for transferring the contents of the product register to the setting levers, which correspond to the keyboard in electric machines. This lends itself directly to the formation of $a b c$ or abcde ... without intermediate settings-a convenience that often outweighs the advantage of keyboard setting or electric drive. The application to $(a \pm b \pm c \cdots) x$ or $(a b \pm c d \pm e f \cdots) x$ is obvious.

The operation $\frac{a}{b c}$ is performed by forming $b c$, transferring, and then using build-up division-exclusively a hand or non-automatic machine processto produce $a$ in the product register and the desired quotient in the multiplier register. Again, a negative result such as ..999 384192 in the product register may be transferred; a backward turn then produces 615808 , which may then, if desired, be transferred to the setting levers to act as a multiplicand. It is difficult to exaggerate the usefulness of this feature, which I commend to the attention of future designers. It is sometimes combined with another feature, namely the ability to clear half the product register without disturbing the other half. Products formed in the righthand portion of the product register may be transferred to the setting levers and, after a carriage movement, added or subtracted in the (temporarily) non-clearing left-hand or storage portion. This permits the formation of $\Sigma a b$ (regardless of signs) with the examination of each individual product; the same is true of $\Sigma a b c$. Again, it permits summation from second differences without any intermediate writing or setting. Another application is the formation in one operation of moments $\Sigma f x$ and $\Sigma f x^{2}$ from equidistant values of $x$.

The multiplier register of this machine has a unique device that enables it to show white figures for positive multiplications and red figures (with full carry over or tens transmission) for negative multiplications or for quotients in tear-down division. The great virtue of this device is that it has no lever, but is operated by the direction of the first turn after a clearance of the multiplier register - a backward turn slides a window and exposes the red figures.

Model 20 (circa 1933) of this machine has the large capacity $12 \times 11 \times 20$. The great advantage of 12 setting levers is that the machine lends itself to doing two small multiplications simultaneously, since there is room to separate the two multiplicands. An earlier variant (Model III) had two multiplier registers, one for individual multipliers or quotients, and one for their sum. Still another, called the Brunsviga Dupla (1928), with capacity $15 \times 10 \times 15$, had two product registers, one of which could be disconnected at will by a lever, and had white and red figures as in the multiplier register. The contents of either register could be transferred to the setting levers. This machine was used for summation from second differences, or for differencing a function directly to its second difference with a single setting of each function value. From a series of positive values of $x$ and $y$, it could form $\Sigma x, \Sigma y, \Sigma x^{2}, \Sigma x y, \Sigma y^{2}$ and check them in one run.

About 1930 there appeared a twin Brunsviga, made by connecting two $10 \times 8 \times 13$ machines so that they are driven by a common handle. The 
left-hand machine can be turned in the same direction as the right-hand machine, or in the opposite direction, or it can be disengaged altogether from the drive. The obvious application is to the simultaneous multiplication of two multiplicands by the same multiplier; for example by setting $\sin \theta$ and $\cos \theta$ and multiplying by $r$, we get $x=r \sin \theta$ and $y=r \cos \theta$ together, one on each machine. With small 3-figure numbers four products can be formed together, i.e. the twins become quads. An expression of the form $\frac{a b}{c}$ can be evaluated in one operation; while the right-hand machine is forming $\frac{a}{c}$, the left-hand machine, which is rotating in the opposite direction, and on which $b$ is set, is forming the product $b \times \frac{a}{c}$.

The original application of twin machines was to military rectangular co-ordinate survey; during the war intensive use was made of them for this purpose by both sides. Since the position of each point is defined by two co-ordinates, it is easily realised that techniques for determining $x$ and $y$ simultaneously can be developed. As an excellent illustration, suppose we know the co-ordinates of two points $A$ and $B$ and the bearings $\alpha$ and $\beta$ to an unknown point $P$, whose co-ordinates are required; this is the intersection problem-the most fundamental in surveying. It is easily shown that

$$
\begin{aligned}
x_{P} & =x_{A}-y_{A} \tan \alpha+y_{P} \tan \alpha \\
& =x_{B}-y_{B} \tan \beta+y_{P} \tan \beta
\end{aligned}
$$

If a value of $y_{P}$ can be found such that the two expressions on the right-hand side are equal to each other, they must both be equal to $x_{P}$. Hence one machine is devoted to the upper equation and one to the lower. After entering $x_{A}$ and $x_{B}$ in the two product registers, $\tan \alpha$ is set on its machine and multiplied by $-y_{A}$. This machine is then disengaged temporarily while $-y_{B} \tan \beta$ is added to the other product register. The two machines, which now have different quantities in their product registers, are once more connected, and the handle turned until they are balanced. Both product registers then show $x_{P}$ and the multiplier register shows $y_{P}$.

Twin machines have also been applied successfully to the interpolation of pairs of double-entry tables, such as those giving rectangular co-ordinates with geographical co-ordinates (latitude and longitude) as argument. The inversion of such pairs, in order to interchange argument and respondent, is another achievement. All this points to their future use for making and interpolating tables of functions of complex variables-a problem in which numerical inversion may often be easier than analytical inversion.

During the war, when Brunsvigas could not be obtained, an attempt was made to twin the one and only British-made machine of this type-the Britannic, a copy of the Brunsviga produced 30 years ago during the first world war. The machine itself proved too unreliable for military conditions, so the problem was solved by twinning (in England) lease-lend hand Marchants, which proved entirely satisfactory. Thus it has come about that there are hundreds of twin Marchants in England, but none in U. S. A., where the single machines were born!

In the Facit machine lever setting is replaced by setting from a $10-\mathrm{key}$ keyboard. This leads to an attractive, compact and inexpensive machine of 
capacity $10 \times 10 \times 19$, at less than half the cost of an electric machine. The advantages of electric drive appear to me to be over-estimated; for many purposes I would prefer two hand machines to a single electric machine, especially when we remember that, with short-cutting in multiplication, a hand machine takes only 60 per cent of the number of revolutions taken by an automatic machine. The real advantage of an electric machine lies not so much in the lessening of muscular effort as in the facilities (if provided) for storage, transfer, accumulation, and automatic division.

The Mercedes Model $38 \mathrm{MSL}$, of capacity $8 \times 8 \times 16$, is a good example of a highly-developed electric calculating machine. Multiplication and division are automatic; products and multipliers can both be accumulated with full and independent control over signs. Any sum or product can become the multiplier of the next operation. There is a visible storage register, which can receive from or transfer to the product register. The two last-named facilities lend themselves immediately to the formation of $\Sigma a b c$.

The electric Madas is the third machine capable of doing $\Sigma a b c$ without intermediate settings. Its peculiar form of automatic multiplication lends itself to forming $\Sigma a$ and $\Sigma a^{2}$ with a single setting of each value of $a$. If two small numbers $a$ and $b$ are set, the machine will produce $\Sigma a, \Sigma b, \Sigma a^{2}, 2 \Sigma a b$ and $\Sigma b^{2}$ in one run-a delightful feature that should be appreciated by those who dabble in correlations. This facility also appeared later on the Monroe Model AA-1-213, which has also followed the German Hamann Selecta in incorporating automatic short-cutting. A useful and unique feature of this Monroe is the ability to call a stored constant multiplier at will instead of setting it when required; this is applicable to $x+a y$ where $x$ and $y$ are variable and $a$ is constant.

Although trying to avoid favouritism, I must confess my admiration for the principle of the automatic multiplication of the American Marchant. In all other automatic machines, both multiplicand and multiplier must be set before multiplication actively begins; the fastest machines run at about $600-700$ revolutions a minute. In the Marchant the multiplicand is set on the keyboard as usual, but the multiplier is entered by an auxiliary row of ten keys. As each key is pressed, its digit is absorbed in to the multiplier and the carriage moved to the next position. While the machine is running on one digit, the next may be set. As the speed is equivalent to 1300 revolutions a minute, the product is always completed within half a second of the setting of the last digit of the multiplier. The high speed mentioned is possible because the numeral wheels are driven by continuous gears (selected by the keyboard setting) rather than engaged and disengaged for a fraction of a revolution according to the digit set.

The American Friden has followed the German Rheinmetall (sold in England as the Muldivo and in U. S. A. as the Mathematon) in adopting a 10-key set-up for the multiplier in automatic multiplication. However these machines do not have complete tens transmission in their product registers -a defect that militates against their use for scientific work.

There is today no desk calculating machine on the market that multiplies directly rather than by repeated addition. Early in the century we had the Swiss Millionaire, but it is no longer manufactured. For each digit of the multiplier it made two rather slow internal strokes-one for the tens and one for the units of the partial products. It could not compete today 
in speed with the Marchant. Moreover it was not convenient for division, as each digit of the quotient had to be estimated before the machine was operated; errors, which could easily arise in borderline cases, were troublesome to correct.

We have now achieved satisfactory speed in multiplication. In future developments of commercial machines we may look, not for electronic speeds, but for the removal of the bottleneck of recording results. Not only is this process time-consuming, but it is a source of error. In the late 1920's there appeared the United machine, with separate keyboards for multiplier and multiplicand, and short-cut multiplication. In three seconds a $6 \times 6$ product could be formed and printed, as well as its two factors. Unfortunately it did not survive the depression that followed its appearance, and is today only to be seen in a Powers multiplying punch, so that the need for an office desk machine that computes and prints is still with us.

The ease with which modern machines can form sums of products has led, in U. S. A. at any rate, to a revival of the Lagrange interpolation formula.

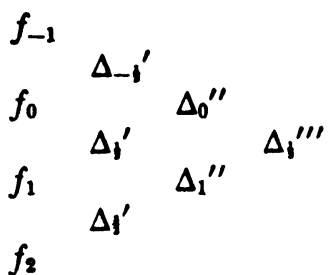

$$
\begin{aligned}
& \text { Instead of using tabular values and their } \\
& \text { differences, an interpolate } f_{n} \text { lying a fraction } \\
& n \text { of the way between } f_{0} \text { and } f_{1} \text { is formed by } \\
& \text { multiplying several tabular values by appro- } \\
& \text { priate weights. }
\end{aligned}
$$

Thus, for a so-called 4-point formula,

$$
f_{n}=L_{-1}^{i v} f_{-1}+L_{0}^{i v} f_{0}+L_{1}^{i v} f_{1}+L_{2}^{i v} f_{2}
$$

where the $L$ 's (whose sum is always 1 ) are polynomials in $n$. In 1928 I stated that the principal objections to this formula were that the computer was never sure how many tabular values to use, and was powerless to detect an error in any one of them. These have been largely overcome by duplicating each calculation with different coefficients and tabular values. Thus if there were no discrepancy between two values of $f_{n}$, one formed from $f_{-1}, f_{0}$ and $f_{1}$ and the other from $f_{0}, f_{1}$ and $f_{2}$, we could accept them as correct. My objection today is that the method ceases to be convenient if the $L$ 's are not directly tabulated; even the most extensive tables available do not have a finer interval than $\mathbf{0 . 0 0 0 1}$. Nevertheless Lagrangean interpolation is a more useful tool today than it was twenty years ago, and lends itself well to the fast tape-controlled special machines now being developed.

At this stage I would like to include an illustration of technique in applying the machines that have been mentioned. It is to inverse interpolation. If $y=f(x)$ is tabulated at equal intervals of $x$, with its differences, and if we require $x$ corresponding to a given value of $y$, we must, theoretically, solve an equation of the second, third or fourth order.

Taking Bessel's formula in the form

where

$$
f_{n}=f_{0}+n \Delta_{i}^{\prime}+B^{\prime \prime}\left(M_{0}^{\prime \prime}+M_{1}^{\prime \prime}\right)+B^{\prime \prime \prime} \Delta_{i}^{\prime \prime \prime}
$$

$$
\begin{gathered}
B^{\prime \prime}=\frac{n(n-1)}{4} \quad B^{\prime \prime \prime}=\frac{n(n-1)\left(n-\frac{1}{2}\right)}{6} \\
M^{\prime \prime}=\Delta^{\prime \prime}-0.184 \Delta^{i v}
\end{gathered}
$$


(see the description of the throw-back given by Mr. Womersley on page 111f), we may rewrite the formula as

$$
f_{n}-B^{\prime \prime}\left(M_{0}^{\prime \prime}+M_{1}^{\prime \prime}\right)-B^{\prime \prime \prime} \Delta_{i}^{\prime \prime \prime}=f_{0}+n \Delta_{i}^{\prime}
$$

in which $n$ and the $B$ 's (which depend on $n$ ) are all unknown. If we can find a value of $n$ such that, when substituted in $B^{\prime \prime}$ and $B^{\prime \prime \prime}$, the two sides of this equation balance, we shall have solved our problem. Two machines are used, one for each side; our object is to balance them. With $f_{n}$ in the lefthand machine we first ignore the second and third order terms, set $f_{0}$ in the right-hand product register and $\Delta_{i}^{\prime}$ on the levers; after balancing the product registers, the right-hand multiplier register shows the $n$ that would result from linear inverse interpolation. This is usually sufficient to determine $B^{\prime \prime \prime}$ finally, so the term $-B^{\prime \prime \prime} \Delta_{t}^{\prime \prime \prime}$ is added on the left-hand machine; then $M_{0}^{\prime \prime}+M_{1}^{\prime \prime}$ is set on the levers and multiplied by the value of $B^{\prime \prime}$, derived (preferably from a critical table) with the approximate value of $\boldsymbol{n}$. This upsets the balance, which must be restored by turning the right-hand machine; the alteration in $n$ will, in general, alter $B^{\prime \prime}$ slightly, and destroy the balance again. This iteration process is continued until there is a static balance with $B^{\prime \prime}$ on the left-hand machine, corresponding to $n$ on the right. In systematic work our computers make a rough allowance for the term $B^{\prime \prime}\left(M_{0}^{\prime \prime}+M_{1}^{\prime \prime}\right)$ from the beginning, and play a "game" with themselves that the first value of $B^{\prime \prime}$ used shall not differ by more than one unit in the last decimal from the final value. Hence inverse interpolation need no longer be shirked, but just taken in the computer's stride. But just a word of warning: do not try this process with automatic machines; hand or semiautomatic machines are needed.

We now turn to a completely different field-that of multi-register machines and their application to problems in finite differences. BABBAGE's efforts more than a century ago to construct a difference engine were frustrated by an enemy that is still with us-apathy and lack of understanding by the administrative lords. However, he inspired ScHEUTz who succeeded in completing a model-now in a museum in Chicago-of which an English copy, after a brief active life, also found its way to a museum at South Kensington. Other spasmodic attempts produced further museum exhibits. In 1914 T. C. Hudson, of the Nautical Almanaic Office, used a printing Burroughs machine, with repeated settings, for numerical integration. It was on this machine in 1928 that I produced 50,000 7-figure values of (then) new functions for the 4-figure tables compiled in collaboration with Milne-Thomson. About this same time we obtained a new Burroughs with an additional register, that could integrate directly from second differences. This machine in 1931 produced $1,200,000$ function values, including those of the 8-figure tables of the four principal trigonometrical functions at interval $1^{\prime \prime}$ that were published by Peters in Berlin in 1939. Copy was also made for a similar 7-figure table; it is now, as far as I know, collecting dust in a Government Department, while less convenient tables, like those of Hof, have been appearing.

This Burroughs triumph was short-lived, for in 1931 the National (really an accounting machine) was "discovered". In its present form it contains six registers, into any combination of which a number can be entered from the keyboard. The contents of any register can be printed, and at the same 
time transferred directly to any combination of the remaining registers. It is this direct transfer that is the secret of the success of this machine for scientific work. In the Burroughs, Continental and Sundstrand machines transfer can be effected only through an intermediary known as the crossfooter, and usually requires various idle or spacing strokes; this, in general, trebles the operating time, and is not offset even by the automatic operation sometimes provided-particularly if the latter can only be changed by a service mechanic.

The principal application of the National machine has been to the differencing of functions to their fifth differences, and to summation from sixth finite differences. The former process is used for checking, and for providing differences for interpolation. The latter occurs extensively in subtabulation, or the systematic breaking down of tables to smaller intervals. It is not too much to claim that this machine has altered our whole attitude to table-making, and has induced a willingness to produce tables that would have been considered too extensive or laborious fifteen years ago. The means of checking values at regular intervals of an independent variable by differencing has relieved modern computing teams of much labour and anxiety and leaves little excuse for error. The 2000 errors marked in our copy of HAYASH's 1926 tables were all found by differencing-and might well have been found in this way by the author himself.

In the work of solving differential equations, multi-register machines go on from where differential analysers leave off. Even the most elaborate and costly analysers can hardly produce more than four significant figures, but there are occasions when more are required. Once we revert to step-by-step summation, machines that will add, subtract, print and store intermediate results come to our aid. It is the storage requirement (for various orders of differences) that calls for many registers-all we have now, and those we may expect in the future. I am confident that the next decade will see a great advance in our treatment of differential equations by numerical integration, and in the evaluation of definite integrals by quadrature.

Finally we come to the punched-card machines, which really fall in the multi-register group. They are distinguished by the fact that they receive their data from cards in which numbers are represented by punched holes; this enables them to fork with great speed. When full advantage can be taken of the system, it is possible to add anything up to 100 figures a second. There are two rival machines of this type. One is electrical and in England (and formerly in U. S. A.) is still called, after its original inventor, the Hollerith; in America it seems to be called now the IBM, after its manufacturing company, International Business Machines. The other machine is mechanical, named after its inventor, Powers. For scientific work the flexibility given by easily-changed electrical connections has been in favour of the Hollerith; the Powers, although well established as an accounting machine, has made little inroad into technical computing.

There are several members of the Hollerith family. Besides machines for punching the holes in cards and checking and reading them, there is a sorter which arranges cards in numerical order. The main machine is the tabulator, with, say, six or eight adding mechanisms; it can print the contents of cards, add and subtract them in groups, and print the totals. The English Hollerith can transfer a number in any register positively or nega- 
tively to any combination of the other registers, but will not subtract directly from a card-a serious drawback. The IBM, on the other hand, will subtract from a card, but is less developed in its transfer facilities. Neither machine is, unfortunately, above reproach in reliability or accuracy-a point to which their designing engineers could well afford to give more attention.

The first scientific application of the Hollerith-in 1928-was to the summation of harmonic terms, or, in other words, Fourier synthesis. In this way the principal terms in the motion of the Moon from 1935 to 2000 were computed. The ordinates of the various harmonics to be compounded were taken from E. W. BRown's Tables of the Moon, and punched on to half a million cards. These were brought together in ever-differing groups; because the periods of the different harmonics are incommensurable, no card ever had the same partners twice, although it may have been used 20 times in the 65 years covered. Something like 100 million figures were added in groups and group totals printed in the course of seven months. I showed this to Brown in the summer of 1928; he had done a great deal of this synthesis himself by hand, and I shall ever remember his ecstasies of rapture as he saw his figures being added at the rate of 20 or 30 a second. I think I am right in saying that the enthusiasm with which he described the process on his return first stimulated W. J. EckERT, the leading American pioneer of these machines for scientific calculations.

In 1932, when the Hollerith first introduced inter-register transfer, it became a rival to the then newly-discovered National for differencing and integrating. That it has not been used more (till recently) for this type of work is probably due to the fact that its rental is such that it must not be allowed any idle moments; its working speed is so great that there may easily be difficulty in producing a continuous supply of work for it. In 1944 we used it to subtabulate to fifths in each direction a large $5 \times 5$ grid of ballistic tables that we had prepared for the U. S. Air Forces. The million figures in the final results were so beautifully printed that photostatic copies met all requirements. It may be noted that whereas the National builds up one difference at a time, the Hollerith can take advantage of the method proposed by Babbage and incorporated by ScHEUTz - that of integrating in two strokes, in one of which even differences are added to odd, while in the other odd differences are added to even. It is not difficult to produce 1000 values an hour by summation; this is several times as fast as a $\mathrm{Na}$ tional, but is not necessarily more economical, as the annual rental of a Hollerith is comparable with the initial purchase price of a National.

Another Hollerith machine-the multiplying punch-can sense numbers $a, b, c, d$ on a card, form combinations like $a b+c \pm d$, and punch them on the same card. In 1933 this was used for extensive conversion of spherical to rectangular co-ordinates. Its use was also suggested by the author for forming apparent places of stars from equations of the type

$$
\alpha=\alpha_{0}+A a+B b+C c+D d
$$

where the capital letters represent quantities that are constant for all stars for any one date, and the small letters represent quantities that are constant for any one star for all dates. At that time this work was divided between four countries, none of which could justify a Hollerith installation, 
but war conditions have led to the application of this method more recently. In preparing The 1940 Heat Drop Tables 2000 initial quantities were first punched on cards, and from these, with the aid of reproducer punches, the multiplier punch, the sorter and the tabulator 66,000 values of the heat drop were produced in a few weeks in the form of copy for press.

Towards the end of the war an IBM machine was employed in England on Fourier synthesis. In the course of the determination of crystal structure by $\mathrm{X}$-ray analysis, it becomes necessary to synthesise the electron density over the volume of a unit cell from expressions of the type

$$
\rho=\sum_{h=0}^{h-H} \sum_{k=0}^{k-K} \sum_{l=0}^{l-L} F_{h k l} \cos 2 \pi h x \cos 2 \pi k y \cos 2 \pi l s
$$

where $H, K$ and $L$ are integers not exceeding $40(10,20,35$ could be considered representative), $F_{h k l}$ are coefficients derived from observation and $x, y, z$ are arbitrary uniform divisions of the cell edges. If in a typical case $x$ and $y$ extend to 30 and $z$ to 60 , we may require to evaluate this expression for $30 \times 30 \times 60=54,000$ points, each of which involves many multiplications and additions; the labour of hand work becomes prohibitive. Various transformations suggested by BEEvERS and LIPSON reduce the work considerably, and their scheme has now been mechanised by having a stack of "master" cards, from which those required for any synthesis are selected (mechanically), reproduced" (again mechanically) on to "slave" or "detail" cards, which are added, and the results printed. The master cards are returned to stock for future use. The cards extend only to the point where $2 \pi x=\frac{1}{2} \pi$; by slight changes of plugging four runs extend the range to $2 \pi$. Provision is made for an interval of $1 / 120$ in $x$. The successful work still being done on salts of penicillin leads to the hope that a complete bureau will be established to relieve crystallographers of their heaviest burden.

The solution of simultaneous linear equations is so laborious that MALLOCK and WILBUR have each made machines for the purpose. Mallock's machine (only one has been made) is in use by the Mathematical Laboratory of Cambridge University; Wilbur's is in storage at M.I.T. What is needed is a machine that is not limited to about ten unknowns, as up to that point the labour of using electric automatic machines is not excessive. A recent study by H. O. HARTLEy (not yet published) of the problem of many unknowns has led to a Hollerith tabulator (not multiplying punch) technique that has proved successful in its early trials. Success in being able to solve large numbers of simultaneous equations readily, whether by an existing machine or otherwise, will confer a great boon on mathematicians, and enable new fields to be explored.

I am convinced that the day of the desk machine is not yet over, or even threatened by the half dozen or so large and special machines that have come into being during the war. Nevertheless I join with others in admiring these machines, and, after seeing so much binary multiplication, feel that LEWIS CARROL should be alive now to write Alice in Onederland. There is however much more to be done before the usefulness to science of the commercial machine is exhausted. There is still room for development in application by Scientific Computing Service, the National Physical Laboratory, the M.I.T. Centre of Analysis, the Watson Computing Labora- 
tory, the New York Mathematical Tables Project, the U. S. Naval Observatory, and others. Let us hear from each of those some report of their ". . . other aids to computation."

L. J. C.

'My youthful typist once typed "mater" cards; perhaps she thought they were better fitted for reproducing.

\section{Publications of the Author}

"Computing by calculating machines," Accountants' J., v. 45, 1927, p. 42-51.

"Recent developments in calculating machines," Office Machinery Users' Assoc., Trans., 1927-28, p. 30-36.

"On the application of the Brunsviga-Dupla calculating machine to double summation with finite differences," R.A.S., Mo. Notices, v. 88, 1928, p. 447-459.

"On the construction of tables by interpolation," R.A.S., Mo. Notices, v. 88, 1928,

p. 506-523.

"Modern Babbage machines," Office Machinery Users' Assoc., Trans., 1931-32, p. 29.

"The Nautical Almanac Office Burroughs machine," R.A.S., Mo. Notices, v. 92, 1932, p. 523-541.

"The application of the Hollerith tabulating machine to Brown's Tables of the Moon,"

R.A.S., Mo. Notices, v. 92, 1932, p. 694-707.

"Computing the Nautical Almanac," Nautical Mag., 1933, 16 p.

The Hollerith and Powers Tabulating Machines, Printed for private circulation, London, 1933, $48 \mathrm{p}$.

Articles "Adding Machines" and "Calculating Machines" in Hutchinson's Technical and Scientific Encyclopaedia, London, 1934.

"Inverse interpolation" and "Scientific applications of the National Accounting

Machine," R. Statistical So., J., v. 3, 1936, suppl., p. 87-114.

"Interpolation and allied tables"; reprinted from the Nautical Almanac for 1937,

p. 784-809, 839, 926-943. Published by H.M. Stationery Office.

"The application of the Brunsviga Twin $13 Z$ calculating machine to the Hartmann

formula for the reduction of prismatic spectograms," The Observatory, v. 60, 1937, p. 70-73.

(With G. B. HEY and H. G. HuDson.) "The application of Hollerith equipment to an

agricultural investigation," R. Statistical So., J., v. 4, 1937, suppl., p. 210-224.

On the application of the Brunsviga Twin $13 Z$ calculating machine to artillery survey, London, Scientific Computing Service, 1938, 18 p.

"Calculating Machines," an Appendix to L. R. Connor, Statistics in Theory and Practice, third ed., London, Pitman, 1938, p. 349-371. 252.

"The use of calculating machines in ray tracing," Phys. So., Proc., v. 52, 1940, p. 246-

The Twin Marchant Calculating Machine and its Application to Survey Problems, London, Scientific Computing Service, 1942, 40 p.

"Mechanical computing," Appendix I to David Clark, Plane and Geodetic Surveying, third rev. ed., v. 2, London, 1944, p. 462-473.

"Careers for girls," Math. Gazette, v. 28, 1944, p. 90-95.

135.

\section{RECENT MATHEMATICAL TABLES}

316[A-D].-Franklin Marion TurRell, Tables of Surfaces and Volumes of Spheres and of Prolate and Oblate Spheroids, and Spheroidal Coefficients. Berkeley and Los Angeles, University of California Press, 1946. xxxiv, 153 p. Offset print. $13.9 \times 21.6 \mathrm{~cm} . \$ 2.00$.

The author of this work is an assistant plant physiologist at the Citrus Experiment Station of the University of California. He tells us that it is necessary to find the areas and volumes of such fruits as lemons, grapefruit, melons, nuts, etc. The lemon is regarded as approximating to a prolate spheroid, and a grapefruit to an oblate spheroid. Assuming that $2 a$ and $2 b$ are the axes of the ellipse of revolution and $\delta=2 a-2 b, \rho=2 b / 2 a, e=$ eccentricity, there are the following tables:

Table 1 (p. 1-3): Surfaces and Volumes of Spheres, $\delta=0, d$ (diameter) $=1(.1) 15$, $3 S$ up to $d=5.6$, then integral values to the end. 